\title{
Perfil cognitivo e funcional de idosos frequentadores dos Centros de Convivência para idosos da cidade de Santos, SP
}

\author{
Nathalia Barros de Andrade ${ }^{\mathrm{a}}$, Marcia Maria Pires Camargo Novelli ${ }^{\mathrm{b}}$ \\ aPrograma de Mestrado Interdisciplinar em Ciências da Saúde, Universidade Federal de São Paulo - UNIFESP, \\ Santos, SP, Brasil

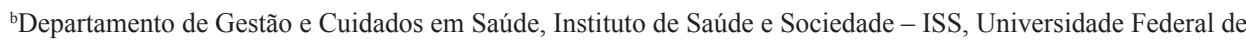 \\ São Paulo - UNIFESP, Campus Baixada Santista, Santos, SP, Brasil
}

\begin{abstract}
Resumo: Introdução: $\mathrm{O}$ crescente aumento da população deve ser acompanhado pela promoção do envelhecimento saudável e a manutenção da máxima capacidade funcional. Objetivo: Identificar o perfil cognitivo e funcional dos idosos que frequentam os Centros de Convivência para terceira idade (Ceconvs) de Santos. Método: Foram avaliados 90 idosos, através dos instrumentos: Questionário de Perfil Sociodemográfico, Questionário Socioeconômico, Mini Exame do Estado Mental (MEEM), Questionário de Queixas Subjetivas de Memória (MAC-Q), Escala de Independência em Atividades da Vida Diária (Escala de Katz) e Escala de Atividades Instrumentais da Vida Diária de Lawton e Brody. Resultados: A média de idade dos idosos foi de 72,5 anos, o gênero predominante foi o feminino (95,5 \%). Houve a predominância de participantes com 1 a 4 anos de escolaridade e a classe econômica dominante foi a classe C. Nas pontuações do MEEM, 63,33\% dos participantes estavam abaixo da nota de corte e no MAC-Q, 71,11\% dos idosos apresentaram disfunções subjetivas de memória. Em relação à funcionalidade, nas Atividades Básicas da Vida Diária (ABVD), 84,44\% dos participantes são independentes. Já para as Atividades Instrumentais da Vida Diária (AIVD), apenas 33,33\% dos participantes são independentes. Nas análises estatísticas entre o perfil cognitivo e de desempenho funcional, foram encontradas associações entre o MEEM e o MAC-Q, MEEM e a Escala de Lawton e Brody e MAC-Q e a Escala de Lawton e Brody. Conclusão: Parte significativa dos idosos frequentadores dos Ceconvs da cidade de Santos apresentam declínios funcionais para atividades básicas e instrumentais da vida diária e declínios cognitivos.
\end{abstract}

Palavras-chave: Terapia Ocupacional, Envelhecimento, Promoção da Saúde, Capacidade Funcional, Cognição.

\section{The Cognitive and Functional Profile of the elderly that frequent the Community Center for the Elderly in the municipality of Santos, Sao Paulo state}

\begin{abstract}
Introduction: The growing population must be accompanied by the promotion of healthy aging and maintain maximum functional capacity. Objective: To understand the cognitive and functional profile of the elderly who take part in the Community Center for the Elderly in Santos, Sao Paulo state. Method: Ninety elderly people were evaluated in this study. The following instruments were used: Social-demographic profile Questionnaire, Socialeconomic Questionnaire, Mini Mental State Examination (MMSE), Subjective Memory Complaint Questionnaire (SMC-Q), Independence in Activities of Daily Living Scale (Katz Scale), and Instrumental Activities of Daily Living Scale (Lawton \& Brody). Results: The average age of the elderly was 72.5 years old and the predominant gender was female (95.5\%). There was a preponderance of participants from 1 to 4 years of education, and Class $\mathrm{C}$ was the predominant economic class. In the MMSE scores, $63.33 \%$ of the participants were below of the cutoff established for education, and in the SMC-Q, $71.11 \%$ of the elderly presented subjective dysfunction of memory. In
\end{abstract}

Autor para correspondência: Nathalia Barros de Andrade, Universidade Federal de São Paulo, Rua Silva Jardim, 136, Vila Mathias, CEP 11015-020, Santos, SP, Brasil, e-mail: nathaliaba@gmail.com

Recebido em Maio 9, 2012; $1^{\text {a }}$ Revisão em Fev. 21, 2013; 2ª Revisão em Mar. 25, 2013; Aceito em Mar. 27, 2013 
the performance of Basic Activities of Daily Living (BADL), $84.44 \%$ of the participants are independent. As for the Instrumental Activities of Daily Living (IADL), only $33.33 \%$ of the participants are independent. In the statistical analyses between cognitive profile and functional performance, associations were found between MMSE and SMC-Q, MMSE and Lawton \& Brody Scale, and SMC-Q and Lawton \& Brody Scale. Conclusions: A significant portion of the elderly patrons of the Community Center presents functional decline and cognitive decline.

Keywords: Occupational Therapy, Aging, Health Promotion, Functional Status, Cognition.

\section{Introdução}

Atualmente, no Brasil, os idosos representam 21 milhóes de pessoas, sendo, portanto $11,3 \%$ da população geral (INSTITUTO..., 2010). Essa população encontra-se principalmente na região Sudeste do país, apresentando maior concentração nos estados do Rio de Janeiro, com 15\% da população idosa, seguido do estado do Rio Grande do Sul, com 13,7\%, e dos estados de São Paulo e Minas Gerais, ambos apresentando 12,2\% da população com 60 anos ou mais (INSTITUTO..., 2009).

No que se refere à cidade de Santos, podemos identificar um grande contingente de pessoas com 60 anos ou mais. A cidade apresenta 19,1\% da população com essa faixa etária, ou seja, 81.423 habitantes da cidade são idosos, sendo assim, o município conta com recursos específicos para essa população na área da saúde, assistência social, esporte e cultura (PREFEITURA..., 2013).

O crescente aumento da população idosa deve ser acompanhado pela melhoria ou manutenção da saúde e qualidade de vida sendo, assim, um desafio para a saúde pública no Brasil. A promoção do envelhecimento saudável e a manutenção da máxima capacidade funcional do indivíduo que envelhece, pelo maior tempo possível, são diretrizes da Política Nacional de Saúde do Idoso (PNSI). Por essa política, capacidade funcional é definida como a capacidade de manter as habilidades físicas e mentais necessárias para uma vida independente e autônoma (BRASIL, 1999).

Os termos independência e a autonomia possuem várias definiçóes na literatura. Segundo a OMS, podemos definir autonomia como a habilidade de controlar, lidar e tomar decisóes pessoais sobre como se deve viver diariamente, de acordo com suas próprias regras e preferências, e independência, como a habilidade de executar funçóes relacionadas à vida diária, isto é, a capacidade de viver independentemente na comunidade com alguma ou nenhuma ajuda de outros. Assim procuramos obter a manutenção da autonomia e o máximo de independência possível visando à melhoria da qualidade de vida (ORGANIZAÇÃO..., 2005).

A capacidade funcional do indivíduo que envelhece é mensurada por meio do grau de dificuldade que o idoso tem ao executar as atividades básicas da vida diária (ABVD) e as atividades instrumentais da vida diária (AIVD) (ORGANIZAÇÃO..., 2005). As ABVD envolvem atividades que relacionam o indivíduo com ele mesmo, por exemplo, tomar banho, vestir-se, comer, dormir e locomover-se pelos cômodos da casa. As AIVD incluem atividades como fazer compras, pagar contas, preparar alimentos, fazer uso de medicamentos, administrar finanças, cuidar da limpeza da casa, usar transportes e telefone, entre outras. As AIVD são consideradas atividades com maior nível de complexidade quando comparadas às ABVD (MELLO; MANCINI, 2007).

Outro grupo de atividades da vida diária são as Atividades Avançadas da Vida Diária (AAVD), que envolvem tarefas cotidianas mais complexas do que as atividades básicas e instrumentais de vida diária e incluem domínios inter-relacionados de atividades de lazer, atividades produtivas e sociais. Essas atividades não são incluídas de forma sistemática na avaliação funcional do idoso devido à necessidade de melhor definição e difusão do termo, a partir da caracterização das atividades incluídas no conjunto das AAVD (DIAS et al., 2011).

Segundo Crepeau (2002), para a execução das atividades da vida diária são requeridas funçóes que na Terapia Ocupacional (TO) são chamadas de componentes de desempenho, como percepção sensorial, propriocepção, equilíbrio, estereognosia, relaçóes espaciais, percepção de profundidade, memória, atenção, capacidade para resolução de problemas, entre outras. A cognição é identificada como um dos componentes de desempenho considerado essencial para a realização das atividades da vida diária, nos permitindo receber o estímulo, processar as informaçóes e consequentemente produzir as respostas (CREPEAU, 2002).

Uma revisão bibliográfica realizada por Royall et al. (2007) verificou que apesar da relevância clínica da relação entre medidas cognitivas e funcionalidade, poucos estudos 
investigam a relação entre elas. Segundo Yassuda (2006), a presença de um declínio gradual da memória tem associação direta com a autonomia e independência do idoso.

$\mathrm{Na}$ rede de serviços públicos propostos para atender a população idosa na cidade de Santos temos os Centros de Convivência para a Terceira Idade (Ceconvs), unidades da prefeitura de Santos gerenciadas pela Secretaria de Assistência Social (SEAS). Os Ceconvs são espaços idealizados para prevenir o isolamento social, com atividades esportivas, artísticas e culturais, entre elas expressão corporal, cursos de idiomas, teatro, dança, cinema, artesanato, bordado, pintura, tapeçaria, aulas de música instrumental, ginástica, ioga, bailes, passeios, caminhadas temáticas, palestras, jogos e encontros da terceira idade. Tais atividades estimulam e preservam o bem-estar físico e emocional dessa população, valorizando a convivência, as relações familiares e comunitárias (PREFEITURA..., 2013).

Segundo a portaria n. 73 do SEAS/MPAS do Ministério da Previdência e Assistência Social, de maio de 2001, o atendimento em centros de convivência consiste no fortalecimento de atividades associativas, produtivas e promocionais, contribuindo para autonomia, envelhecimento ativo e saudável, prevenção do isolamento social, socialização e aumento da renda própria. $\mathrm{O}$ espaço é destinado à frequência dos idosos e de seus familiares, onde são desenvolvidas planejadas e sistematizadas açôes de atenção ao idoso, de forma a elevar a qualidade de vida, promover a participação, a convivência social, a cidadania e a integraçáo intergeracional (BRASIL, 2001).

Diante da notável transição epidemiológica e das problemáticas que envolvem o processo de envelhecimento populacional, cria-se a necessidade de avaliaçóes sobre as alteraçóes do envelhecimento e suas consequências, além de discussóes sobre a rede de serviços para o atendimento dessa populaçáo. Com isso, o presente estudo teve por objetivo caracterizar o perfil sociodemográfico, cognitivo e funcional dos idosos que frequentam os centros de convivência para a terceira idade na cidade de Santos e verificar a existência de associação entre os aspectos da cogniçáo e do desempenho funcional.

\section{Procedimentos metodológicos}

\subsection{Amostra}

Este estudo foi desenvolvido na cidade de Santos, nos Centros de Convivência para a Terceira Idade, em parceria entre a Secretária de
Assistência Social (SEAS) e o curso de Terapia Ocupacional da Universidade Federal de São Paulo (Unifesp) - Campus Baixada Santista. Tanto o projeto como o termo de consentimento livre e esclarecido foram aprovados no Núcleo de Bioética da Unifesp sob número NBBS0110_09.

Foram avaliados 90 idosos selecionados de forma semialeatória, a partir de um convite para participação no estudo. Para ser incluído no estudo, o participante deveria ter idade igual ou superior a 60 anos e ser frequentador de um dos Ceconvs da cidade de Santos. Santos conta com três Ceconvs estrategicamente localizados em regiōes diferentes da cidade, sendo assim foram avaliados 30 idosos em cada Ceconv da cidade, a saber: Centro de Convivência "Vida Nova", Centro de Convivência "Isabel Garcia” e Centro de Convivência "Zona Noroeste".

Em relação aos aspectos éticos do estudo, as avaliaçóes só foram aplicadas nos idosos a partir do esclarecimento dos objetivos do estudo e de os idosos terem consentido participar, com a assinatura do termo de consentimento livre e esclarecido. Os dados foram coletados junto aos idosos de setembro a dezembro de 2009.

\subsection{Instrumentos de avaliação}

Os instrumentos utilizados, adaptados para a cultura brasileira, confiáveis e validados, foram aplicados por meio de entrevistas. O protocolo completo contém seis instrumentos e levou em média 45 minutos para aplicação.

Para descrição do perfil sociodemográfico dos idosos foram utilizados: o questionário de perfil sociodemográfico elaborado pela pesquisadora, que contempla dados de identificação do participante e informações sociodemográficas como nome, data de nascimento, idade, endereço, telefone para contato, gênero e escolaridade. Para a avaliação socioeconômica optou-se pelo critério da Associação Brasileira dos Institutos de Pesquisa de Mercado (Abipeme), que classifica a populaçáo por meio de cinco classes, denominadas A, B, C, D e E (ALMEIDA; WICKERHAUSER, 1991).

Os aspectos cognitivos foram avaliados com os instrumentos: Mini Exame do Estado Mental (MEEM) (FOLSTEIN; FOLSTEIN; MCHUGH, 1975) e Questionário de Queixas Subjetivas de Memória (MAC-Q) (CROOK; FEHER; LARABEE, 1992).

O MEEM é um teste de rastreio de comprometimento cognitivo que fornece uma 
pontuação geral variando de 0 a 30 pontos, sendo a escolaridade o fator demográfico de maior importância na determinação da pontuação final. A nota de corte utilizada foi: 20 pontos (analfabetos), 25 pontos ( 1 a 4 anos de escolaridade), 26,5 pontos ( 5 a 8 anos), 28 pontos ( 9 a 11 anos) e 29 pontos ( $\geq$ a 11 anos). Optou-se pela utilizaçáo dessa pontuação para a uniformização dos resultados, por sua boa aplicabilidade em ambientes hospitalares e ambulatoriaia e em estudos populacionais (FOLSTEIN; FOLSTEIN; MCHUGH, 1975, BRUCKI et al., 2003).

O MAC-Q é constituído por cinco perguntas que avaliam diferentes aspectos de memória relacionados a situaçóes do cotidiano, as respostas variam de "muito pior agora" a "muito melhor agora", em uma escala likert de 5 pontos. A pontuação no teste pode variar de 7 até 35 pontos, sendo a pontuaçáo máxima relacionada com máxima percepção subjetiva de disfunção na memória. Considera-se pontuaçáo igual ou superior a 25 indicativa de disfunção subjetiva de memória (CROOK; FEHER; LARABEE, 1992, MATTOS et al., 2003, YASSUDA et al., 2006).

Os aspectos funcionais foram avaliados com os instrumentos: Escala de Independência em Atividades da Vida Diária (Escala de Katz) e Escala de Atividades Instrumentais da Vida Diária de Lawton e Brody. A Escala de Katz é amplamente utilizada para avaliar o desempenho do idoso nas atividades básicas da vida diária, baseia-se em funçôes primárias biológicas e psicossociais e contém seis itens que medem o desempenho do indivíduo nas atividades de autocuidado: alimentação, controle de esfíncteres, transferência, higiene pessoal, capacidade para se vestir e tomar banho (LINO et al., 2008).

A Escala de Atividades Instrumentais da Vida Diária de Lawton e Brody foi empregada com o propósito de identificar a condiçâao funcional do idoso na realizaçáo de AIVD. Essa escala apresenta sete domínios: uso do telefone, viagens, realização de compras, preparo das refeiçóes, trabalho doméstico, uso de medicamentos e manuseio do dinheiro. Sua pontuação varia de 7 a 21 e, quanto menor o valor obtido pelo idoso, maior o grau de comprometimento para levar uma vida independente (SANTOS; VIRTUOSO, 2008).

\subsection{Análise estatística}

Os dados foram digitados em planilhas do programa Excel 2007 para Microsoft Windows $\mathrm{XP}$ e foram analisados por meio do programa estatístico SPSS, versão 17.0. Foram feitas análises descritivas das características sociodemográficas dos participantes. Os resultados foram apresentados em tabelas de frequência. No que tange à variável idade foram feitas estimativas das medidas de tendência central e de dispersão. As associaçóes entre as variáveis numéricas foram estudadas e estabelecidas utilizando-se o coeficiente de correlação de Pearson e, para o estudo e estabelecimento de associaçóes entre as variáveis numéricas e categóricas, foi utilizada a análise de variância com fator fixo (Oneway ANOVA) e o teste $\mathrm{t}$ - student para amostras não relacionadas (Independent - Samples T test). O grau de significância foi fixado para valores de $p<0,05$.

\section{Resultados}

\subsection{Descrição do perfil sociodemográfico dos idosos}

A caracterizaçáo sociodemográfica e socioeconômica dos participantes, está descrita na Tabela 1 .

\subsection{Caracterização do perfil cognitivo dos idosos}

A Tabela 2 apresenta o desempenho dos participantes no MEEM de acordo com as notas de corte propostas por Brucki et al. (2003) e os resultados da aplicação do MAC-Q, de acordo com a nota de corte para indicativo de disfunção subjetiva de memória proposta por Crook, Feher e Larabee (1992).

Os itens do MEEM que apresentaram pior desempenho foram a realização de cálculos, seguida da memória de evocação e construção do desenho. Entre as questôes do MAC-Q, observamos que a questão 6 (Descrição global da memória) apresentou maior média de queixas subjetivas de memória, seguida pela questáo 3 (Lembrar onde colocou objetos) e, em terceiro lugar, pela questão 1 (Lembrar o nome de pessoas que acabou de conhecer).

\subsection{Caracterização do perfil de desempenho funcional dos idosos}

Para a avaliação do desempenho funcional dos participantes nas AVD foi utilizada a Escala de ABVD de Katz e a Escala de AIVD de Lawton 
e Brody. A Tabela 3 apresenta os resultados relacionados ao desempenho dos participantes nas ABVD e AIVD.

Os resultados indicam que $84,44 \%$ dos idosos são independentes em todas as seis funçóes da escala (tomar banho, vestir-se, uso do vaso sanitário, transferência, continência e alimentação) e 15,55\% dos idosos apresentam dificuldades relacionadas à continência. Os itens que apresentaram maior nível de dependência na Escala de Lawton e Brody

Tabela 1. Perfil sociodemográfico e socioeconômico.

\begin{tabular}{lcccc}
\hline \multicolumn{1}{c}{ Características } & Ceconv VN & Ceconv IG & Ceconv ZN & Total \\
\hline Idade & & & & \\
Mínimo & 61 & 62 & 61 & 61 \\
Máximo & 93 & 89 & 85 & 93 \\
Média & 72,36 & 72,96 & 72,40 & 72,54 \\
Desvio padrão & 7,16 & 6,59 & 6,30 & 6,62 \\
Gênero & & & & \\
Feminino & $100 \%$ & $86,6 \%$ & $100 \%$ & $95,5 \%$ \\
Masculino & $0 \%$ & $13,3 \%$ & $0 \%$ & $4,4 \%$ \\
Escolaridade & & & & \\
Analfabetos & $3,3 \%$ & $3,3 \%$ & $6,6 \%$ & $4,4 \%$ \\
1 a 4 anos & $36,6 \%$ & $66,6 \%$ & $90 \%$ & $64,4 \%$ \\
5 a 8 anos & $40 \%$ & $23,3 \%$ & $3,3 \%$ & $22,2 \%$ \\
9 a 11 anos & $6,6 \%$ & $0 \%$ & $0 \%$ & $2,2 \%$ \\
+ de 11 anos & $4,4 \%$ & $6,6 \%$ & $0 \%$ & $6,6 \%$ \\
Econômica & & & & \\
Classe A & $3,33 \%$ & $0 \%$ & $0 \%$ & $1,11 \%$ \\
Classe B & $16,6 \%$ & $10 \%$ & $3,33 \%$ & $10 \%$ \\
Classe C & $53,33 \%$ & $56,66 \%$ & $56,66 \%$ & $55,55 \%$ \\
Classe D & $20 \%$ & $26,66 \%$ & $30 \%$ & $25,55 \%$ \\
Classe E & $6,66 \%$ & $6,66 \%$ & $10 \%$ & $7,77 \%$ \\
\hline VN & & & & \\
\hline
\end{tabular}

VN - Vida Nova; IG - Isabel Garcia; ZN - Zona Noroeste.

Tabela 2. Perfil cognitivo dos participantes.

\begin{tabular}{lcccc}
\hline \multicolumn{1}{c}{ Instrumentos } & Ceconv VN & Ceconv IG & Ceconv ZN & Total \\
\hline MEEM & & & & \\
Acima & $30 \%$ & $33,33 \%$ & $23,33 \%$ & $28,88 \%$ \\
Na média & $3,33 \%$ & $10 \%$ & $10 \%$ & $7,77 \%$ \\
Abaixo & $66,66 \%$ & $56,66 \%$ & $66,66 \%$ & $63,33 \%$ \\
MAC-Q & & & & \\
Com indicativo & $60 \%$ & $70 \%$ & $83,33 \%$ & $71,11 \%$ \\
Sem indicativo & $40 \%$ & $30 \%$ & $16,66 \%$ & $28,88 \%$ \\
\hline
\end{tabular}

VN - Vida Nova; IG - Isabel Garcia; ZN - Zona Noroeste; MEEM - Mini Exame do Estado Mental; MAC-Q - Questionário de Queixas Subjetivas de Memória.

Tabela 3. Desempenho funcional.

\begin{tabular}{lcccc}
\hline \multicolumn{1}{c}{ Instrumentos } & Ceconv VN & Ceconv IG & Ceconv ZN & Total \\
\hline ABVD & & & & \\
Independência & $86,66 \%$ & $80 \%$ & $86,66 \%$ & $84,44 \%$ \\
Dependência & $13,33 \%$ & $20 \%$ & $13,33 \%$ & $15,55 \%$ \\
AIVD & & & & \\
Independência & $36,66 \%$ & $20 \%$ & $43,44 \%$ & $33,33 \%$ \\
Dependência & $63,33 \%$ & $80 \%$ & $56,66 \%$ & $66,66 \%$ \\
\hline
\end{tabular}

VN - Vida Nova; IG - Isabel Garcia; ZN - Zona Noroeste; ABVD - Atividades Básicas da Vida Diária; AIVD - Atividades Instrumentais de Vida Diária. 
foram a realização de trabalho doméstico $(52,22 \%)$, seguida pela realização de viagens $(27,77 \%)$ e, posteriormente a administração de medicamentos $(13,33 \%)$ e o manuseio do dinheiro $(12,22 \%)$.

\subsection{Correlação entre os aspectos da cognição e do desempenho funcional}

A Tabela 4 apresenta os coeficientes de correlação entre o MEEM, MAC-Q e Escala de AIVD de Lawton e Brody.

$\mathrm{Na}$ análise de correlação entre o MEEM, MAC-Q e a Escala de Lawton e Brody, foi encontrada associação entre o MEEM e MAC-Q, entre o MEEM e a Escala de Lawton e Brody e entre o MAC-Q e a Escala de Lawton e Brody.

A Tabela 5 apresenta os coeficientes de correlação entre os instrumentos MEEM, MAC-Q e Escala Lawton e Brody com a Escala de Katz.

$\mathrm{Na}$ análise de correlação entre a Escala de Katz com os instrumentos MEEM, MAC-Q e a Escala de Lawton e Brody não foram encontradas associaçóes significativas.

\section{Discussão}

A proporção de pessoas com 60 anos ou mais apresenta um crescimento mundial mais rápido do que o das outras faixas etárias. Sendo assim, o envelhecimento populacional é gerado principalmente pela reduçáo da taxa de mortalidade e aumento da expectativa de vida (ORGANIZAÇÃO..., 2005).
Atualmente, o aumento da expectativa de vida está vinculado ao aumento de doenças crônicodegenerativas e das limitaçôes funcionais, sendo necessário, segundo a OMS, a implementação de políticas e programas orientados pela política "envelhecimento ativo", que melhorem a saúde, a participação e a segurança dos idosos. Novas medidas para manter a populaçáo idosa saudável e ativa são uma necessidade em todo o mundo (ORGANIZAÇÃO..., 2005).

A média de idade dos idosos frequentadores dos três Ceconvs da cidade de Santos foi de 72,54 anos. Em relação ao gênero, os resultados indicam que 95,5\% dos participantes são do gênero feminino e 4,4\% do gênero masculino. A predominância feminina entre os idosos pode ser explicada em parte pela menor taxa de mortalidade da população feminina, o que pode ser atribuído a fatores biológicos e à diferença de exposição aos fatores de risco de mortalidade (PAPÁLEO NETTO, 2006).

$\mathrm{O}$ número elevado de mulheres participantes da pesquisa, além de se relacionar com o processo de feminização da velhice, pode também demonstrar uma maior participação das mulheres em atividades fora do ambiente domiciliar. As mulheres, mais do que os homens, participam de atividades extradomésticas, de organizaçóes e movimentos de mulheres, cursos, viagens, trabalhos voluntários e trabalhos remunerados temporários (CAMARANO, 2006).

Em relação à escolaridade, $64,4 \%$ dos participantes possui de 1 a 4 anos de estudo. Segundo o Censo de 2000, na última década houve um aumento significativo no percentual de idosos

Tabela 4. Coeficientes de correlação entre as pontuações do MEEM, MAC-Q e Escala de Lawton e Brody.

\begin{tabular}{lccc}
\hline & MEEM & MAC-Q & Escala de Lawton e Brody \\
\hline MEEM & & & \\
Person & ------- & $-0,320 * *$ & $0,252^{*}$ \\
Sig. & & 0,002 & 0,017 \\
MAC-Q & & & \\
Pearson & $-0,320 * *$ & $-----282^{* *}$ \\
Sig. & 0,002 & & 0,007 \\
\hline
\end{tabular}

**Correlação significativa ao nível 0,01; *Correlação significativa ao nível 0,05.

Tabela 5. Coeficientes de correlação entre a Escala de Katz e o MEEM, MAC-Q e Escala de Lawton e Brody.

\begin{tabular}{lccc}
\hline & MEEM & MAC-Q & Escala Lawton e Brody \\
\hline $\begin{array}{l}\text { Escala de Katz } \\
\text { Test-T }\end{array}$ & & & \\
p. & 0,812 & 0,339 & 0,348 \\
\hline
\end{tabular}


alfabetizados no país mas, apesar dos avanços, ainda existem 5,1 milhôes de idosos analfabetos. Em relação à influência do gênero sobre a escolaridade, os homens continuam sendo, proporcionalmente, mais alfabetizados do que as mulheres, $67,7 \%$ contra $62,6 \%$, respectivamente, já que até os anos 60 tinham mais acesso à escola do que as mulheres (INSTITUTO..., 2002).

$\mathrm{Na}$ avaliação socioeconômica, a classe $\mathrm{C}$ apresentou maior porcentagem entre os participantes $(55,5 \%)$, seguida da Classe D $(25,5 \%)$. Foi identificado que o Ceconv Zona Noroeste apresentou o menor nível socioeconômico, seguido do Ceconv Isabel Garcia e, por último, encontramos o Ceconv Vida Nova com o maior nível socioeconômico.

Segundo dados obtidos no site da prefeitura de Santos, o Ceconv Zona Noroeste está localizado numa das regiôes mais carentes da cidade de Santos, justificando o menor nível socioeconômico e a menor escolaridade dos idosos desse Ceconv. O Ceconv Vida Nova, localizado próximo da praia, em uma zona nobre da cidade, apresentou o maior nível socioeconômico e o maior nível de escolaridade. Já o Ceconv Isabel Garcia apresentou níveis socioeconômicos e de escolaridade entre os níveis apresentados pelos dois outros Ceconvs citados anteriormente. Ele está localizado no bairro da Encruzilhada, que se encontra no meio da chamada Santos Leste e é considerado um bairro de classe média (PREFEITURA..., 2013).

A Fundação Perseu Abramo (2007) realizou uma pesquisa sobre o perfil sociodemográfico dos idosos brasileiros em abril de 2006, em 204 municípios distribuídos pelas cinco regiôes do país (Norte, Nordeste, Sul, Sudeste e Centro-Oeste). Verificou-se que $43 \%$ da população idosa têm renda familiar de até 2 salários-mínimos e que apenas 3\% recebem acima de 10 salários. Além disso, de acordo com o mesmo estudo, identificou-se que a população idosa é mais urbana, mais feminina e menos escolarizada. De acordo com essa pesquisa, $89 \%$ dos participantes não passaram da oitava série do ensino fundamental e $18 \%$ não tiveram educação formal.

Estudo realizado por Valin-Ragatto, Candalo e Bretas (2011) com uma amostra de 291 idosos frequentadores de centros de convivência na cidade de Cuiabá (MT, Brasil) demonstrou que a maior porcentagem de idosos da amostra possuia de 65 a 69 anos, era do gênero feminino, tinha de 1 a 4 anos de escolaridade e recebia em torno de 1 a 2 salários-mínimos. Tais dados corroboram os dados sociodemográficos encontrados por este estudo.
Em relação ao perfil cognitivo, identificamos por meio do MEEM que 63,3\% dos participantes apresentaram pontuaçóes abaixo das notas de corte propostas para a escolaridade descritas por Brucki et al., 2003. Tais resultados apontam para a presença de declínio cognitivo objetivo dos idosos participantes dos Centros de Convivência.

Os itens do MEEM que apresentaram pior desempenho foram a realização de cálculos, seguida da memória de evocaçáo e construçáo do desenho. Segundo Hartman-Maeir, Katz e Baum (2009), na avaliação de domínios cognitivos específicos, especial atenção deve ser dada a atenção, memória e funçóes executivas, sendo esses componentes essenciais para a realização das AVD.

Com o aumento da idade, algumas funçóes cognitivas se mantêm estáveis e outras declinam. As funções que sofrem maior impacto por efeito do passar dos anos são as relacionadas ao raciocínio abstrato (p. ex., cálculo), a aprendizagem de material inédito, a nomeação de objetos, as habilidades visuoespaciais e a velocidade no processamento das informaçôes (KIRSHNER, 2002).

Através do MAC-Q identificamos as queixas subjetivas de disfunção de memória presentes entre os idosos participantes da pesquisa, observando que $71,1 \%$ dos idosos apresentaram pontuaçóes que indicam disfunção subjetiva de memória. Segundo Jonker, Geerlings e Schmand (2000), a idade avançada, o sexo feminino e um baixo nível de escolaridade estáo associados à alta prevalência de queixas de memória.

A queixa de esquecimento é tópico importante no campo da Geriatria, devendo ser investigada e avaliada tanto na clínica quanto com o uso de instrumentos adequados. Uma queixa de esquecimento pode ser um sinalizador da instalação de declínio cognitivo ou de quadros mais importantes, como as demências. Estudo realizado na Zona Norte do Rio de Janeiro com idosos participantes de um centro de convivência identificou que $44,2 \%$ dos participantes relataram problemas de esquecimentos (VERAS et al., 2007).

No estudo foram encontradas correlações entre as duas escalas cognitivas utilizadas, MEEM e MAC-Q $(r=-0,320 ; p<0,01)$, demonstrando associação entre o desempenho cognitivo objetivo avaliado por meio do MEEM e as queixas subjetivas de memória avaliadas através MAC-Q.

Segundo a OMS (ORGANIZAÇÃO..., 2005), o conceito de funcionalidade é fator preponderante para o diagnóstico de saúde física e mental da populaçáo idosa. A avaliação funcional dos 
participantes foi realizada especificamente para atividades básicas e instrumentais por meio da Escala de Independência nas Atividades da Vida Diária (Escala de Katz) e da Escala de Atividades Instrumentais da Vida Diária desenvolvida por Lawton e Brody. Essas atividades têm associação mais frequente com a autonomia e independência do idoso.

A avaliação funcional dos idosos no estudo não incluiu a realização das AAVD que englobam aspectos de qualidade de vida, trabalho e lazer, por não haver disponível, na literatura, instrumentos validados para a avaliação dessas atividades. Segundo Dias et al. (2011), apesar da menção da inclusão das AAVD na avaliação geriátrica abrangente, não foram descritos instrumentos validados específicos para sua avaliaçáo.

Os resultados encontrados em relaçáo às ABVD, na Escala de Katz, apresentaram que as pontuaçóes dos idosos participantes indicam independência em cinco das seis funçóes. A única função comprometida foi a continência, ou seja, 15,5\% dos participantes relataram a ocorrência de acidentes ocasionais de controle da micção e/ou evacuaçáo. Dentre as alteraçóes comuns com o processo de envelhecimento encontramos a incontinência urinária que, além de possuir múltiplas etiologias, com grande complexidade terapêutica, gera um enorme impacto sobre a qualidade de vida dessas pessoas. A incontinência urinária não é uma alteração inerente ao processo de envelhecimento, porém há uma alta incidência na população de idosos (HONORIO; SANTOS, 2009).

A partir dos resultados obtidos na Escala de AIVD de Lawton e Brody identificamos que $66,6 \%$ dos idosos apresentam dependência parcial nessas atividades. As atividades que apresentaram maior nível de dependência para a realização foram: trabalho doméstico, seguido da realizaçáo de viagens, administração de medicamentos e manuseio do dinheiro.

Segundo Nygard (2003), as AIVD são habilidades altamente complexas e demandam grande capacidade de julgamento, raciocínio e planejamento, sendo mais vulneráveis ao declínio cognitivo. Em um estudo que investigava as AVD em idosos brasileiros residentes da cidade de Goiânia foi encontrado que as atividades para as quais os idosos apresentavam maior dependência parcial foram manuseio de dinheiro, uso do transporte e trabalho doméstico (COSTA; NAKATANI; BACHION, 2006).
$\mathrm{Na}$ análise de correlação entre os aspectos da cognição e do desempenho funcional não foram encontradas correlaçóes entre a Escala de Katz e os instrumentos cognitivos, porém foi possível identificar correlaçôes entre as pontuaçôes totais do MEEM com as pontuaçôes totais da Escala de Lawton e Brody $(r=0,252 ; p<0,05)$. Foi encontrada também associação entre as pontuaçôes totais do MAC-Q com as pontuaçôes totais da Escala de Lawton e Brody $(r=-0,282 ; \mathrm{p}<0,01)$, demonstrando a associação entre cognição e funcionalidade. Segundo Perneczky et al. (2006), as atividades instrumentais da vida diária (AIVD) são dependentes do funcionamento cognitivo intacto, considerando memória, atenção e funções executivas, e estão susceptíveis a leves declínios cognitivos, tornando-se comprometidas.

As limitaçóes encontradas no estudo estáo relacionadas ao fato de a amostra utilizada ser pequena e não representativa da população idosa como um todo e à pesquisa ter sido realizada nos centros de convivência de uma cidade específica. Sendo assim, não se pode afirmar que o perfil cognitivo e funcional encontrado refira o de idosos frequentadores de centros de convivência para idosos de outras regióes.

\section{Conclusão}

Com base nos resultados podemos concluir que os idosos participantes desta amostra, frequentadores dos Centros de Convivência da cidade de Santos apresentam declínios funcionais para atividades básicas e instrumentais da vida diária e declínios cognitivos. Os problemas de cognição identificados estão nos seguintes domínios: cálculo, memória de evocação e construção do desenho. As queixas de memória estão presentes em maior proporção no desempenho global da memória e em situaçóes como lembrar onde colocaram objetos e lembrar o nome de pessoas que acabaram de conhecer. Já os problemas de desempenho funcional identificados relacionam-se à continência (a ocorrência de acidentes ocasionais de controle da micção e/ou evacuação), realização de trabalho doméstico, realização de viagens, administração de medicamentos e manuseio de dinheiro. Foi identificado que as alteraçôes cognitivas encontradas associam-se com as alteraçóes funcionais.

Acredita-se que esses resultados subsidiarão profissionais e estudiosos da área da gerontologia na caracterização do perfil sociodemográfico, cognitivo e funcional de idosos frequentadores de centros 
de convivência, primordial para a proposição de atividades e acompanhamento dos participantes.

\section{Referências}

ALMEIDA, P. M.; WICKERHAUSER, H. O critério ABA/ABIPEME: em busca de uma atualização. São Paulo: Associação Brasileira de Anunciantes: Associação Brasileira dos Institutos de Pesquisa de Mercado, 1991. BRASIL. Ministério da Saúde. Portaria no 1395, de 9 de dezembro de 1999. Política Nacional de Saúde do Idoso. Diário Oficial da República Federativa do Brasil. Poder Executivo, Brasília, DF, 13 dez. 1999. n 237-E, Seção 1, p. 20-24.

BRASIL. Secretaria de Estado de Assistência Social. Portaria no 73, de 10 de Maio de 2001. Estabelece normas de funcionamento de serviços de atenção ao idoso no Brasil, nas modalidades previstas na Política Nacional do Idoso, e aos desafios que o crescimento demográfico impóe ao país. Diário Oficial da República Federativa do Brasil. Poder Executivo, Brasília, DF, 14 maio 2001.

BRUCKI, S. M. et al. Sugestôes para o uso do mini-exame do estado mental no Brasil. Arquivos de Neuro-Psiquiatria. Sáo Paulo, v. 61, n. 3B, p. 777-81, 2003. http://dx.doi. org/10.1590/S0004-282X2003000500014

CAMARANO, A. A. Envelhecimento da populaçấo brasileira: Uma contribuição demográfica. In: FREITAS E. V. et al. Tratado de Geriatria e Gerontologia. Rio de Janeiro: Guanabara Koogan, 2006. p. 1162-1169.

COSTA, E. C.; NAKATANI, A. Y. K.; BACHION, M. M. Capacidade de idosos da comunidade para desenvolver atividades de vida diária e atividades instrumentais de vida diária. Acta Paulista de Enfermagem, Goiânia, v. 19, n. 1, p. 43-48, 2006. http://dx.doi.org/10.1590/ S0103-21002006000100007

CREPEAU, E. B. Análise de atividades: uma forma de refletir sobre desempenho ocupacional. In: NEISTADT, M. E.; CREPEAU, E. B. Terapia Ocupacional. Rio de Janeiro: Guanabara Koogan, 2002. p. 121-133.

CROOK, T. H.; FEHER, E. P.; LARABEE, G. J. Assessment of memory complaint in age-associated memory impairment: the MAC-Q. International Psychogeriatric, Bethesda, v. 4, n. 2, p. 165-176, 1992. http://dx.doi.org/10.1017/S1041610292000991

DIAS, E. G. et al. Caracterização das atividades avançadas de vida diária (AAVDS): um estudo de revisão. Revista de Terapia Ocupacional da Universidade de São Paulo, São Paulo, v. 22, n. 1, p. 45-51, 2011.

FOLSTEIN, M. F.; FOLSTEIN, S. E.; MCHUGH, P. R. "Mini-mental state". A practical method for grading the cognitive state of patients for the clinician. Journal of Psychiatric Research, Portland, v. 12, n. 3, p. 189-98, 1975. PMID: 1202204

FUNDAÇÃO PERSEU ABRAMO. Idosos no Brasil vivências, desafios e expectativas na $3^{\mathrm{a}}$ idade. São Paulo, 2007. Disponível em: <http://novo.fpabramo. org.br/content/idosos-no-brasil-vivencias-desafios-eexpectativas-na-3a-idade>. Acesso em: 28 mar. 2012.
HARTMAN-MAEIR, A.; KATZ, N.; BAUM, C. M. Cognitive Functional Evaluation (CFE) process for individuals with suspected cognitive disabilities. Occupational Therapy Health Care, Mount Scopus, Jerusalem, v. 23, n. 1, p. 1-23, 2009. PMID: 23930828. http://dx.doi.org/10.1080/07380570802455516

HONORIO, M. O.; SANTOS, S. M. Incontinência urinária e envelhecimento: Impacto no cotidiano e na qualidade de vida. Revista Brasileira de Enfermagem, Florianópolis, v. 62, n. 1, p. 51-56, 2009.

INSTITUTO BRASILEIRO DE GEOGRAFIA E ESTATÍSTICA - IBGE. Sintese de Indicadores Sociais 2010. Rio de Janeiro, 2010. Disponível em: <http:// www.ibge.gov.br>. Acesso em: 30 jul. 2011.

INSTITUTO BRASILEIRO DE GEOGRAFIA E ESTATISTICA - IBGE. Pesquisa Nacional por Amostra de Domicilios - PNAD 2009. Rio de Janeiro, 2009. Disponível em: <http://www.ibge.gov.br>. Acesso em: 20 fev. 2011.

INSTITUTO BRASILEIRO DE GEOGRAFIA E ESTATISTICA - IBGE. Perfil dos idosos responsáveis pelos domicílios. Rio de Janeiro: Comunicação Social, 2002. Disponível em: <http://www.ibge.gov.br>. Acesso em: 30 jul. 2011.

JONKER, C.; GEERLINGS, M. I.; SCHMAND, B. Are memory complaints predictive for dementia? A review of clinical and population-based studies. Journal of Geriatric Psychiatry, Nederland, v. 15, n. 11, p. 983-991, 2000.

KIRSHNER, H. Behavioral neurology: pratical science of mind and brain. Boston: Butterworth-Heinemann, 2002.

LINO, V. T. S. et al. Adaptação transcultural da escala de independência em atividades da vida diária (Escala Katz). Caderno de Saúde Pública, Rio de Janeiro, v. 24, n. 1, p. 103-112, 2008.

MATTOS, P. et al. Memory complaints and test performance in healthy elderly persons. Arquivos de Neuro-Psiquiatria, São Paulo, v. 61, n. 4, p. 920-924, 2003. http://dx.doi.org/10.1590/S0004-282X2003000600006

MELLO, M. A. R.; MANCINI, M. C. Avaliação das atividades de vida diária e controle domiciliar. In: CAVALCANTI, A.; GALVÃO, C. Terapia ocupacional: fundamentação e prática. Rio de Janeiro: Guanabara Koogan, 2007. p. 49-54.

NYGARD, L. Instrumental activities of daily living: a stepping-stone towards Alzheimer's disease diagnosis in subjects with mild cognitive impairment? Acta Neurologica Scandinavica, Supplementum, Londres, v. 179, p. 42-6, 2003.

ORGANIZAÇÃO MUNDIAL DA SAÚDE - OMS. Envelhecimento Ativo: Uma política de saúde. Brasília: Organização Pan-Americana da Saúde, 2005.

PAPÁlEO NETTO, M. P. O estudo da velhice: Históricos, definição do campo e termos básicos. In: FREITAS, E. V. et al. Tratado de Geriatria e Gerontologia. Rio de Janeiro: Guanabara Koogan, 2006. p. 1-12. 
PERNECZKY, R. et al. Complex activities of daily living in mild cognitive impairment: conceptual and diagnostic issues. Age Ageing, Germany, v. 35, n.3, p. 240-245, 2006. http://www.ncbi.nlm.nih.gov/pubmed/16513677.

PREFEITURA MUNICIPAL DE SANTOS.Cidade@: Santos. Disponível em <www.santos.sp.gov.br>. Acesso em: 18 fev. 2013.

ROYALL, D. R. et al. The cognitive correlates of functional status: A review from the committee on research of the American Neuropsychiatric Association. Journal of Neuropsychiatry and Clinical Neurosciences, Arlington, v. 19, n. 3, p. 249-265, 2007. http://dx.doi. org/10.1176/jnp.2007.19.3.249

SANTOS, R. L.; VIRTUOSO, J. S. J. Confiabilidade da versão brasileira da escala de atividades instrumentais da vida diária. Revista Brasileira em Promoção da Saúde, Bahia, v. 21, n. 4, 2008. p. 290-296.

VALIN-RAGATTO, P. C.; CANDALO, C.; BRETAS, A. C. P. Nível de atividade física e sua relação com quedas acidentais e fatores psicossociais em idosos de Centro de Convivência. Revista Brasileira de Geriatria e Gerontologia, Rio de Janeiro, v. 14, n. 3, p. 521-531, 2011.

VERAS, R. et al. Promovendo a saúde e prevenindo a dependência: Identificando indicadores de fragilidade em idosos independentes. Revista Brasileira de Geriatria e Gerontologia, Rio de Janeiro, v. 10, n. 3, p. 355-370, 2007.

YASSUDA, M. S. Memória e envelhecimento saudável. In: FREITAS, E. V. et al. Tratado de geriatria e gerontologia. Rio de Janeiro: Guanabara Koogan, 2006. p. $1245-1251$.

YASSUDA, M. S. et al. Treino de memória em idosos saudáveis: Mecanismos e benefícios. Psicologia: Reflexão e Crítica, Porto Alegre, v. 19, n. 3, p. 470-481, 2006. http://dx.doi.org/10.1590/ S0102-79722006000300016

\section{Contribuição dos Autores}

Nathalia Barros de Andrade participou de todas as etapas do estudo, incluindo a coleta e análise dos dados, e redação do texto. Marcia Maria Pires Camargo Novelli orientou todas as etapas do estudo, incluindo a coleta, análise dos dados e revisão crítica do texto.

\section{Fonte de Financiamento}

Bolsa de Iniciação Científica do Conselho Nacional de Desenvolvimento Científico e Tecnológico - CNPq. 